\title{
Análisis del cumplimiento de profilaxis antibiótica en colecistectomía laparoscópica electiva en un hospital de México
}

\author{
Analysis of the compliance of antibiotic prophylaxis in elective \\ laparoscopic cholecystectomy in a Mexican hospital

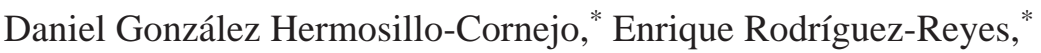 \\ Diego Abelardo Álvarez-Hernández, „ Amado de Jesús Athíe-Athíe, \\ Pablo Andrade-Martínez Garza, ${ }^{*}$ José Manuel Correa-Rovelo*
}

Palabras clave: Colecistectomía, infección asociada a la asistencia sanitaria, infección del sitio quirúrgico, laparoscopia, profilaxis antibiótica preoperatoria.

Keywords: Cholecystectomy, infection associated with healthcare, surgical site infection, laparoscopy, preoperative antibiotic prophylaxis.

\footnotetext{
* Programa de Cirugía General, Facultad Mexicana de Medicina de la Universidad La Salle, México. ‡ Programa de Enfermedades Infecciosas, London School of Hygiene \& Tropical Medicine, Londres, Reino Unido.

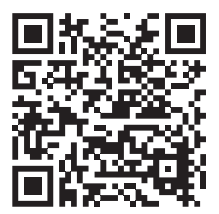

\section{RESUMEN}

Introducción: La administración de profilaxis antibiótica preoperatoria reduce el riesgo de infecciones del sitio quirúrgico; sin embargo, pese a que existen recomendaciones preestablecidas para su uso, éstas con frecuencia no se cumplen. Objetivo: Describir el cumplimiento y resultados de la profilaxis antibiótica preoperatoria en pacientes sometidos a colecistectomía laparoscópica electiva dentro de nuestra institución. Material y métodos: Se realizó un estudio observacional, longitudinal prospectivo y descriptivo que incluyó a los pacientes sometidos a colecistectomía laparoscópica electiva dentro de nuestro hospital del 1 de julio al 31 de diciembre de 2018 en busca del desarrollo de infecciones del sitio quirúrgico y otras complicaciones. Resultados: Al 97\% de los pacientes (n = 162 ) se les administró profilaxis antibiótica preoperatoria, encontrándose una prescripción correcta en sólo $54 \%$ de los casos $(n=87)$. Solamente $1 \%$ de los pacientes $(n=2)$ presentó infección del sitio quirúrgico. Conclusiones: En nuestro estudio, pese a existir una baja prescripción correcta de profilaxis antibiótica preoperatoria, sólo $1 \%$ desarrolló infección del sitio quirúrgico, razón por la cual apoyamos los argumentos de algunas guías de práctica clínica de no prescribirla de manera sistemática, sino seleccionando a los pacientes de acuerdo con sus características y riesgos.

\section{ABSTRACT}

Introduction: The administration of preoperative antibiotic prophylaxis reduces the risk of surgical site recommendations for their prescription, these are often not met. Objective: To describe the compliance and results of preoperative antibiotic prophylaxis in elective laparoscopic cholecystectomy in our center. Material and methods: An observational, longitudinal, prospective and descriptive study was conducted, including the patients who underwent elective laparoscopic cholecystectomy in our hospital from July 1 to December 31 of 2018 in search of surgical site infection and other complications. Results: Preoperative antibiotic prophylaxis was administered in $97 \%(n=162)$ of the cases, but only in $54 \%$ of the patients $(n=87)$ was prescribed correctly. Only $1 \%(n=2)$ presented surgical site infection. Conclusions: In our study, despite there being a low correct prescription of preoperative antibiotic prophylaxis, only $1 \%$ of the cases developed surgical site infection, which is why we support the arguments of some clinical practice guidelines of not prescribing it systematically, but selecting the patients according to their characteristics and risks instead. infection development, however, although there are preset

\section{INTRODUCCIÓN}

$\mathrm{L}^{2}$ as infecciones del sitio quirúrgico (ISQ) se definen como "infecciones relaciona- das con el procedimiento quirúrgico que se producen cerca del sitio de incisión dentro de los primeros 30 días a partir de su realización o dentro de los primeros 90 días a partir
Citar como: González Hermosillo-Cornejo D, Rodríguez-Reyes E, Álvarez-Hernández DA, Athíe-Athíe AJ, Andrade-Martínez Garza P, Correa-Rovelo JM. Análisis del cumplimiento de profilaxis antibiótica en colecistectomía laparoscópica electiva en un hospital de México. Cir Gen. 2020; 42 (4): 274-280. https://dx.doi.org/10.35366/101396 
de la colocación de un implante", ${ }^{1}$ mientras que las infecciones asociadas a la atención sanitaria (IAAS) se definen como "infecciones que adquieren los pacientes mientras reciben atención médica". Las ISQ son las IAAS que se presentan con mayor frecuencia en países

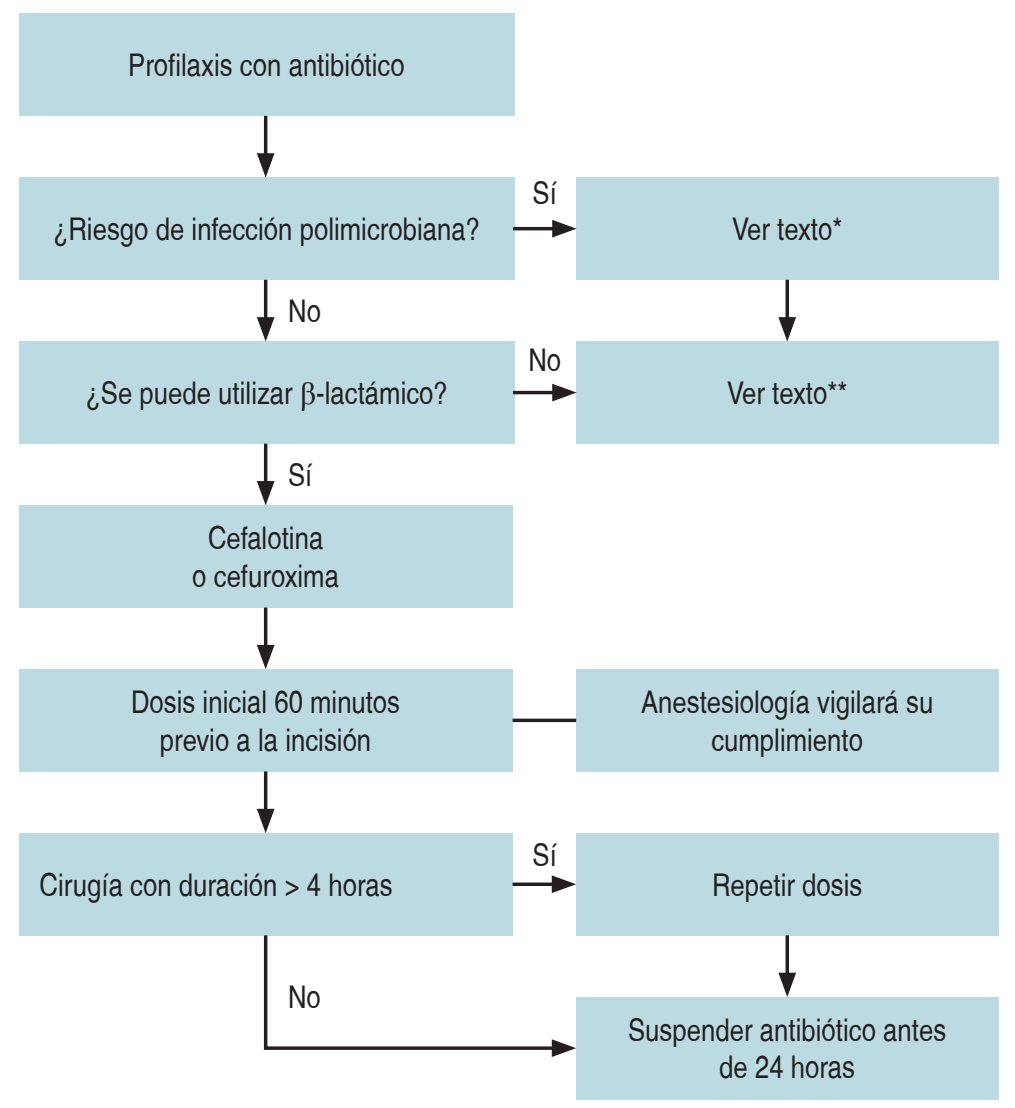

${ }^{*}$ Antibióticos recomendados:

1. Primera elección:

a. Cefalotina 1 gramo vía intravenosa dentro de los 60 minutos previos a la incisión

b. Cefuroxima 1.5 gramos vía intravenosa dentro de los 60 minutos previos a la incisión

\section{Alternativa:}

a. Ampicilina/sulbactam 2 gramos/1 gramo vía intravenosa dentro de los 60 minutos previos a la incisión

b. Cefalotina + metronidazol $500 \mathrm{mg}$ vía intravenosa o clindamicina $600 \mathrm{mg}$ vía intravenosa dentro de los 60 minutos previos a la incisión

** Opción en caso de que se encuentre contraindicado el uso de $\beta$-lactámicos: vancomicina 1 gramo vía intravenosa en infusión dentro de los 120 minutos previos a la incisión

Figura 1: Diagrama de flujo para la profilaxis antibiótica preoperatoria. Diagrama elaborado a partir de nuestra guía hospitalaria para la profilaxis quirúrgica con antibióticos. de ingresos bajos y medios (PIBM), afectando hasta a uno de cada tres pacientes que se someten a procedimientos quirúrgicos $y$, aunque se observan con menor frecuencia en países de ingresos medios y altos (PIMA), aún son el segundo tipo más frecuente de IAAS en Europa y Norteamérica. ${ }^{2}$

La profilaxis antibiótica preoperatoria (PAP) se define como "la prevención de complicaciones infecciosas mediante la administración efectiva de antibióticos previa a la contaminación durante un procedimiento quirúrgico". ${ }^{3}$ Ésta combate la contaminación bacteriana de los tejidos que en condiciones normales se encuentran libres de microorganismos y evita que la flora endógena o exógena que ingresa al área quirúrgica se multiplique y favorezca el desarrollo de la infección. ${ }^{4,5}$ En la gran mayoría de los procedimientos quirúrgicos suele recomendarse la administración efectiva de PAP y, aunque instituciones como la Organización Mundial de la Salud (OMS), el Centro para el Control y la Prevención de Enfermedades (CDC, por sus siglas en inglés), ${ }^{1}$ el Instituto Nacional de Excelencia en Salud y Atención (NICE, por sus siglas en inglés $)^{6}$ y la Sociedad de Enfermedades Infecciosas de América (IDSA, por sus siglas en inglés), ${ }^{3}$ entre otras, han publicado guías de práctica clínica $(\mathrm{GPC})$ con recomendaciones precisas sobre su manejo, se ha demostrado que éstas no se cumplen de manera cotidiana y que la PAP suele administrarse de manera ineficiente y arbitraria. $^{7}$ En nuestro país, el Instituto Mexicano del Seguro Social (IMSS), a través de la GPC "Prevención y diagnóstico de la infección de sitio quirúrgico", es el encargado de emitir las recomendaciones que se aplican de manera específica en nuestro territorio, ${ }^{8} \mathrm{y}$ cada hospital de adaptarlas de acuerdo con su perfil de resistencia antimicrobiana (Figura 1). Apegarnos a ellas al hacer buen uso de los antibióticos disminuye la resistencia antimicrobiana y mejora el pronóstico del paciente. ${ }^{9}$

La colecistectomía laparoscópica electiva (CLE) es el procedimiento abdominal que se realiza con mayor frecuencia a nivel mundial y es el tratamiento de elección para pacientes con colelitiasis y colecistitis aguda. ${ }^{10}$ Debido a esto, se han efectuado múltiples estudios que han presentado resultados controversiales sobre el desarrollo de infecciones en este pro- 
cedimiento. En 2010, Sanabria y colaboradores realizaron una revisión Cochrane que incluyó 11 ensayos clínicos aleatorizados con 1,664 pacientes concluyendo que la evidencia clínica no era suficiente para apoyar o refutar el uso de PAP. ${ }^{11}$ Por otra parte, en 2018, Sajid y su equipo realizaron una revisión sistemática y metaanálisis que incluyó 25 ensayos clínicos aleatorizados con 6,138 pacientes con el mismo objetivo, obteniendo resultados estadísticamente significativos en el grupo control y evidenciando la importancia de una correcta $\mathrm{PAP}^{12}$ por lo que es evidente la necesidad de llevar a cabo nuevos estudios que tengan la capacidad de brindar una respuesta esclarecedora a este problema.

El objetivo de este artículo fue analizar el cumplimiento de la PAP de los pacientes sometidos a CLE en un hospital de tercer nivel de la Ciudad de México.

\section{MATERIAL Y MÉTODOS}

Se realizó un estudio observacional, longitudinal prospectivo y descriptivo, el cual se sometió a revisión por el Comité Hospitalario de Bioética e Investigación de nuestra institución siendo aprobado con el número 2018EXT295. Se revisaron los expedientes clínicos de todos los pacientes que fueron sometidos a CLE dentro de un hospital de tercer nivel en la Ciudad de México durante el periodo comprendido entre el 1 de julio y el 31 de diciembre de 2018 en busca del desarrollo de complicaciones postquirúrgicas.

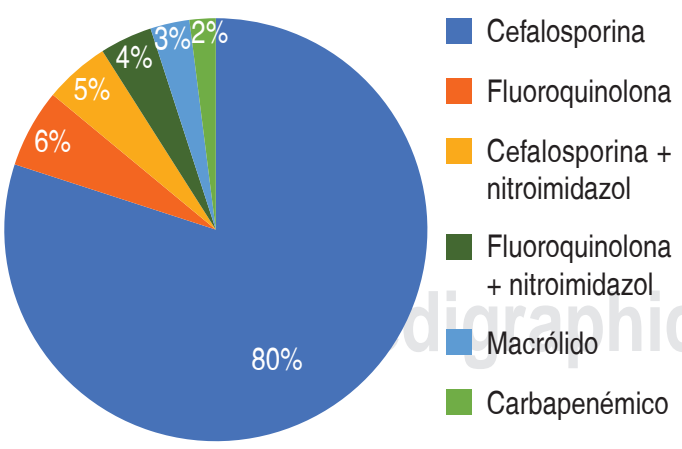

Figura 2: Grupos de antibióticos que se administraron con mayor frecuencia como parte de la profilaxis antibiótica preoperatoria. Figura elaborada a partir de nuestros resultados.

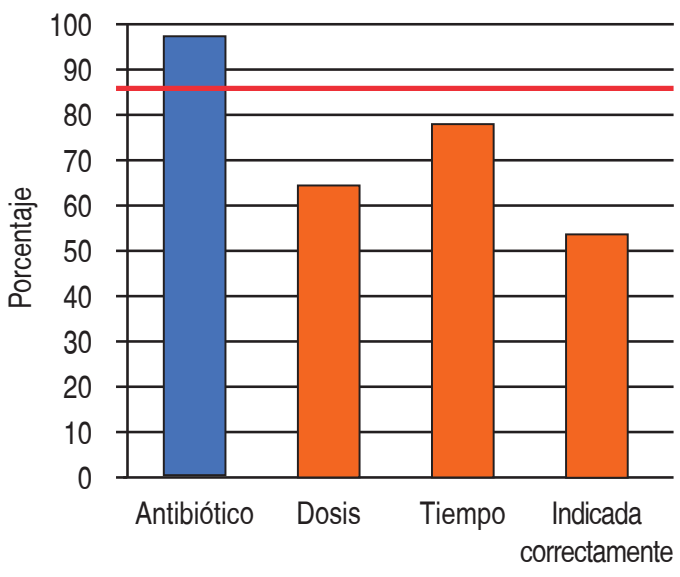

Figura 3: Prescripción de profilaxis antibiótica preoperatoria (antibiótico, dosis y tiempo). Figura elaborada a partir de nuestros resultados.

Se incluyeron todos los pacientes mayores de un año que fueron sometidos a una CLE dentro del tiempo de estudio $(n=227)$, mientras que se excluyeron aquéllos cuyos hallazgos transquirúrgicos condicionaron la administración de antibióticos de manera terapéutica $(n=60)$. No fue necesario eliminar expedientes clínicos por ambigüedad o falta de información. Se consideraron para el análisis 167 expedientes clínicos. Las variables de estudio fueron: género, edad, alergias, comorbilidades (tabaquismo, sobrepeso, obesidad, diabetes tipo 2 e inmunosupresión), diagnóstico, PAP (antibiótico, dosis y tiempo), desarrollo de ISQ, escala ClavienDindo, días de estancia intrahospitalaria (DEIH) y reingreso. Se capturó en formato electrónico la información de las historias clínicas para su análisis estadístico, mismo que se llevó a cabo con el software Statistical Package for the Social Sciences (SPSS) v.24 para obtener medidas de tendencia central y de dispersión.

\section{RESULTADOS}

De los pacientes a los que se les realizó una CLE, $44 \%$ correspondieron al sexo masculino y $56 \%$ al sexo femenino. La edad promedio fue de 48 años (DE \pm 15.36). El diagnóstico de ingreso más común fue colelitiasis en $88 \%$ ( $n=147)$ de los casos, seguido de poliposis con $9 \%(n=15)$, discinesia en $2 \%(n=3)$ y otros $1 \%(n=2)$. 
De los pacientes, $43 \%(n=72)$ refirieron tener tabaquismo activo, mientras que las comorbilidades que se presentaron con mayor frecuencia fueron sobrepeso en $67 \%(n=112)$ de los casos, obesidad en $26 \%(n=43)$, diabetes tipo 2 en $15 \%(n=25)$ e inmunosupresión en $4 \%(n=7)$.

De los pacientes, $14 \%(n=23)$ refirieron ser alérgicos a por lo menos un antibiótico, siendo más común la alergia hacia la penicilina en $57 \%$ $(n=13)$ de los casos.

A $97 \%(n=162)$ de los pacientes se les administró PAP, siendo las cefalosporinas el grupo de antibióticos que se administró con mayor frecuencia en $80 \%(n=130)$ de los casos (Figura 2).

La dosis fue correcta en $65 \%$ de los casos a los que se les indicó antibiótico ( $n=105)$, mientras que el tiempo para una administración adecuada se cumplió en $78 \%$ de los pacientes ( $n=126$ ) (Figura 3); sin embargo, al analizar las tres variables de la PAP (tiempo correcto, antibiótico empleado y posología adecuada), ésta se indicó de manera correcta sólo en 54\% $(n=87)$ de los casos.

De los pacientes, $2 \%(n=4)$ presentaron complicaciones postquirúrgicas: $1 \%(\mathrm{n}=2)$ tuvieron infección del sitio quirúrgico y $1 \%$ (n $=2$ ) otro tipo de complicaciones, mientras que sólo un paciente fue clasificado como grado IV en la escala Clavien-Dindo (anafilaxia).

El tiempo promedio de estancia intrahospitalaria fue de 2.05 días ( $D E \pm 1.22$ ) y fue necesario el reingreso en sólo $2 \%(n=3)$ de los casos.

Adicionalmente, la información de 3\% ( $n=$ 5) de los pacientes a los que se les realizó un
CLE, pero que no se les administró PAP por indicación del cirujano, se muestra en la Tabla 1.

\section{DISCUSIÓN}

Las ISQ y las complicaciones que de ellas se derivan son un problema frecuente y potencialmente letal que supone un incremento importante de la morbilidad y mortalidad, tiempo de estancia hospitalaria y costos sanitarios, ${ }^{13}$ pudiéndose asociar con cualquier tipo de procedimiento quirúrgico. En 2013, el Centro Europeo para el Control y la Prevención de Enfermedades (ECDC, por sus siglas en inglés) realizó un estudio en 16 países donde reportó que la incidencia acumulada más alta de acuerdo al tipo de procedimiento quirúrgico la presentó la cirugía de colon con 9.5 casos por cada 100 operaciones, seguida de la cirugía de revascularización Coronaria con 3.5 casos, cesárea con 2.9 casos y colecistectomía con 1.4 casos. ${ }^{14}$ La frecuencia acumulada de ISQ en CLE en nuestro hospital para el periodo de estudio fue de 1.19 casos por cada 100 operaciones de CLE. En los resultados se menciona que $1 \%$ corresponde a dos pacientes de 167 estudiados, por lo que las cantidades no coinciden, son ligeramente menores que lo reportado en la literatura.

En nuestro estudio, 93\% de los pacientes que se sometieron a CLE presentaron sobrepeso u obesidad, 43\% tabaquismo, $15 \%$ diabetes tipo 2 y $4 \%$ inmunosupresión, incrementando considerablemente su riesgo de desarrollar ISQ.

Investigaciones recientes han contrastado la información mostrada en distintas guías

\section{Tabla 1: Resumen de la historia clínica de los pacientes a los que no se les administró profilaxis antibiótica preoperatoria.}

\begin{tabular}{lccccccccc} 
Paciente & Edad & Sexo & Diagnóstico & Comorbilidad & Alergias & PAP & ISQ & DEIH & Reingreso \\
\hline 1 & 48 & Femenino & Poliposis & Sobrepeso & Negadas & No & No & 2 & No \\
2 & 50 & Masculino & Poliposis & Sobrepeso & Negadas & No & No & 2 & No \\
3 & 29 & Femenino & Colelitiasis & Sobrepeso & Negadas & No & No & 4 & No \\
4 & 43 & Femenino & Colelitiasis & Ninguna & Negadas & No & No & 2 & No \\
5 & 55 & Femenino & Colelitiasis & Sobrepeso y DT2 & Negadas & No & No & 1 & No \\
\hline
\end{tabular}

$\mathrm{PAP}=$ profilaxis antibiótica preoperatoria; ISQ = infección del sitio quirúrgico; DEIH = días de estancia intrahospitalaria; DT2 = diabetes tipo 2. Tabla elaborada a partir de nuestros resultados. 
Tabla 2: Recomendaciones para el uso de profilaxis antibiótica preoperatoria.

\begin{tabular}{|c|c|c|c|}
\hline Tipo de cirugía & Profilaxis recomendada & $\begin{array}{l}\text { Profilaxis recomendada en caso de alergia a } \\
\beta \text {-lactámicos }\end{array}$ & $\begin{array}{l}\text { Grado de } \\
\text { evidencia }\end{array}$ \\
\hline \multicolumn{4}{|l|}{ Laparoscopia: } \\
\hline Electiva (bajo riesgo) & Ninguno & Ninguno & A \\
\hline \multirow[t]{3}{*}{ Electiva (alto riesgo) } & Cefalotina & Clindamicina o vancomicina + aminoglucósido o & A \\
\hline & Ceftriaxona & fluoroquinolona & \\
\hline & Amoxicilina-clavulanato & Metronidazol + aminoglucósido o fluoroquinolona & \\
\hline \multicolumn{4}{|c|}{ 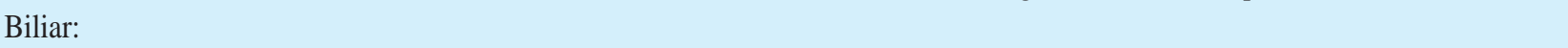 } \\
\hline Procedimiento abierto & Cefalotina & Clindamicina o vancomicina + aminoglucósido o & A \\
\hline & $\begin{array}{l}\text { Ceftriaxona } \\
\text { Amoxicilina-clavulanato }\end{array}$ & fluoroquinolona & \\
\hline Gastroduodenal & Cefalotina & $\begin{array}{l}\text { Clindamicina o vancomicina + aminoglucósido o } \\
\text { fluoroquinolona }\end{array}$ & A \\
\hline \multicolumn{4}{|l|}{ Intestinal: } \\
\hline Con obstrucción & Cefalotina & $\begin{array}{l}\text { Clindamicina o vancomicina + aminoglucósido o } \\
\text { fluoroquinolona }\end{array}$ & $\mathrm{C}$ \\
\hline Sin obstrucción & Cefalotina + metronidazol & Metronidazol + aminoglucósido o fluoroquinolona & $\mathrm{C}$ \\
\hline Apendical* & Cefalotina + metronidazol & $\begin{array}{l}\text { Clindamicina o vancomicina + aminoglucósido o } \\
\text { fluoroquinolona } \\
\text { Metronidazol + aminoglucósido o fluoroquinolona }\end{array}$ & A \\
\hline \multirow[t]{4}{*}{ Colorrectal } & Cefalotina + metronidazol & Clindamicina + aminoglucósido o fluoroquinolona & A \\
\hline & Amoxicilina/clavulanato & Metronidazol + aminoglucósido o fluoroquinolona & \\
\hline & Ceftriaxona + metronidazol & & \\
\hline & Ertapenem & & \\
\hline
\end{tabular}

respecto a los principios establecidos de manera bibliográfica y experimental, denotando el desconocimiento y empleo arbitrario de diversos antibióticos en múltiples centros quirúrgicos. ${ }^{15}$ Como norma general, el tiempo de administración de la PAP debe realizarse una hora previa a la incisión quirúrgica, siendo por lo regular una cefalosporina de primera o de segunda generación el antibiótico de elección (Tabla 2), mientras que como alternativa para pacientes con antecedentes de alergia conocida a la penicilina, se puede administrar vancomicina o clindamicina. ${ }^{8,16}$ Adicionalmente, debe considerarse la evaluación periódica del cuadro epidemiológico y microbiológico de cada institución, la disponibilidad de insumos y las particularidades de las especialidades médicas para determinar la rotación y modificaciones en los antibióticos que serán utilizados. 5,15

En nuestro estudio, a 97\% de los pacientes se les administró PAP, denotando un elevado porcentaje de cumplimiento en este aspecto; sin embargo, se administró la dosis incorrecta en $35 \%$ de los casos sin que se cumplieran las recomendaciones en cuanto al tiempo de aplicación en $22 \%$ de los pacientes, evidenciando la falta de homogeneidad en los criterios aplicados por distintos cirujanos.

Al analizar los resultados de las tres variables que se consideraron para determinar si la prescripción fue correcta (antibiótico, dosis y tiempo), sólo se logró una indicación 
óptima en $56 \%$ de los casos y pese a esto, sólo dos pacientes de la muestra desarroIlaron ISQ.

Respecto a los cinco pacientes que fueron sometidos a CLE, pero que no recibieron PAP por preferencia u omisión del médico tratante, ninguno presentó manifestaciones clínicas compatibles con un proceso infeccioso, su tiempo de estancia intrahospitalaria fue similar al del resto de la población (2.20 vs. 2.05) y en ningún caso fue necesario su reingreso.

En conjunto, tanto la baja incidencia de ISQ, pese a la administración incorrecta de PAP, como la ausencia de ISQ, y la falta de administración de PAP generan controversia sobre si realmente es necesario su uso de manera rutinaria. Se debe considerar que el empleo arbitrario y el abuso de antibióticos tanto en pacientes ambulatorios como hospitalizados se acompaña de un incremento en la aparición de nuevas infecciones, de reacciones adversas y de resistencia antimicrobiana, por lo que la prevención, abordaje y manejo de las ISQ debe ser una actitud activa, continua y primordial para todo el personal relacionado con los cuidados de la salud y especialmente para el cirujano, quien además debe vigilar su posible aparición durante el periodo postoperatorio para que le permita realizar un diagnóstico precoz con el objetivo de proporcionar tratamiento oportuno. ${ }^{13}$

\section{CONCLUSIONES}

En nuestro estudio se cumplió con la prescripción óptima de PAP en aproximadamente la mitad de los casos y a pesar de eso, sólo un mínimo porcentaje de pacientes desarrolló ISQ, razón por la cual apoyamos los argumentos de algunas GPC de no prescribirla de manera sistemática, seleccionando a los pacientes de acuerdo con sus características y riesgos. Sin embargo, consideramos que se requieren estudios con una rigurosa metodología científica para emitir recomendaciones finales. Mientras tanto, concluimos que es imperativo formalizar los programas de actualización continua para homologar los criterios bajo los cuales se administra la PAP así como implementar listas de verificación que orienten a los cirujanos en la toma de decisiones.

\section{REFERENCIAS}

1. Berríos-Torres SI, Umscheid CA, Bratzler DW, Leas B, Stone EC, Kelz RR, et al. Centers for Disease Control and Prevention Guideline for the prevention of surgical site infection, 2017. JAMA Surg. 2017; 152: 784-791. doi: 10.1001/jamasurg.2017.0904.

2. World Health Organization. Global guidelines for the prevention of surgical site infection. Geneva; WHO; 2016. [Consultado en abril de 2019] Disponible en: https://www.who.int/gpsc/ssiguidelines/en

3. Bratzler DW, Dellinger EP, Olsen KM, Perl TM, Auwaerter PG, Bolon MK, et al. Clinical practice guidelines for antimicrobial prophylaxis in surgery. Surg Infect (Larchmt). 2013; 14: 73-156. doi: 10.1089/ sur.2013.9999.

4. Crader MF, Bhimji S. Preoperative antibiotic prophylaxis. In: Bhojani P, Aeby T, Pearson S, et al. StatPearls. Treasure Island: StatPearls Publishing; 2019: 1. Disponible en: https://www.ncbi.nlm.nih.gov/books/ NBK442032/

5. González VJ, González FR, Martínez BML. Antibioticoterapia profiláctica en Cirugía General. Rev Acta Médica. 2011; 13: 83-88.

6. National Institute for Health and Care Excellence. Surgical site infections: prevention and treatment. United Kingdom; NICE; 2018. [Consultado en abril de 2019] Disponible en: https://www.nice.org.uk/ guidance/indevelopment/gid-ng10094

7. Solís-Téllez H, Mondragón-Pinzón EE, RamírezMarino M, Espinoza-López FR, Domínguez-Sosa F, Rubio-Suarez JF, et al. Epidemiologic analysis: prophylaxis and multidrug-resistance in surgery. Rev Gastroenterol Mex. 2017; 82: 115-122. doi: 10.1016/j.rgmx.2016.08.002.

8. Instituto Mexicano del Seguro Social. Prevención y diagnóstico de la infección del sitio quirúrgico. Guía de evidencias y recomendaciones: Guía de Práctica Clínica. México: IMSS; 2018. [Consultado en abril de 2019] Disponible en: http://imss.gob.mx/ profesionales-salud/gpc

9. Charani E, Ahmad R, Tarrant C, Birgand G, Leather A, Mendelson M, et al. Opportunities for system level improvement in antibiotic use across the surgical pathway. Int J Infect Dis. 2017; 60: 29-34. doi: 10.1016/j.ijid.2017.04.020.

10. Coccolini F, Catena F, Pisano M, Gheza F, Fagiuoli S, Di Saverio S, et al. Open versus laparoscopic cholecystectomy in acute cholecystitis. Systematic review and meta-analysis. Int J Surg. 2015; 18: 196204. doi: 10.1016/j.ijsu.2015.04.083.

11. Sanabria A, Dominguez LC, Valdivieso E, Gomez G. Antibiotic prophylaxis for patients undergoing elective laparoscopic cholecystectomy. Cochrane Database Syst Rev. 2010; CD005265. doi: 10.1002/14651858. CD005265.pub2.

12. Sajid MS, Bovis J, Rehman S, Singh KK. Prophylactic antibiotics at the time of elective cholecystectomy are effective in reducing the post-operative infective complications: a systematic review and meta-analysis. Transl Gastroenterol Hepatol. 2018; 3: 22. doi: 10.21037/tgh.2018.04.06. 
13. Santalla A, López-Criado MS, Ruiz MD, Fernández-Parra J, Gallo JL, Montoya F. Infección de la herida quirúrgica. Prevención y tratamiento. Clin Invest Gin Obst. 2007; 34: 189-196. doi: 10.1016/S0210-57X(07)74505-7.

14. European Centre for Disease Prevention and Control. Surveillance of surgical site infections in Europe 20102011. Stockholm: ECDC; 2013 [Consultado en abril de 2019] Disponible en: https://ecdc.europa.eu/en/ publications-data/surveillance-surgical-site-infectionseurope-2010-2011

15. Zubieta OG, González ACA, Cartagena SEJ, Peña VVI, Garzón MJ, Robledo OF. Uso de antibióticos preoperatorios y postoperatorios en el departamento de cirugía general de un hospital privado y comparación con las guías actuales de manejo antimicrobiano. Acta Med. 2016; 14: 12-18.

16. Hansen E, Belden K, Silibovsky R, Vogt M. Antibióticos perioperatorios. Acta Ortop Mex. 2013; 27: 31-59.

\section{Correspondencia:}

Dr. Daniel González Hermosillo-Cornejo

Residencia de Cirugía General.

Puente de Piedra Núm. 150,

Col. Toriello Guerra, 14050,

Tlalpan, Ciudad de México, México.

Teléfono: 55 5424-7200

E-mail: dr.gonzalezhermosillo@gmail.com 